\title{
Project Follow-Up and Change Management Tips
}

\author{
Claudia CARSTEA ${ }^{1}$, Gheorghe SABAU ${ }^{2}$ \\ 1“"George Baritiu” University, Brasov, Romania \\ ${ }^{2}$ Academy of Economic Studies, Bucharest, Romania \\ 15claudiacarstea@gmail.com, sabau@ase.ro
}

The managers have to understand the technological impact and the informatics applications upon the business they lead; taking into account the fact that IT assures them the necessary information. Today's businesses are more and more organized, lead and automatized around the series of processes and the relations established among them. These processes involve developments and the products and services, the interactions with the clients and the support of those processes and of human resources. Thereby, we suggest the identification of the solutions for the successful planning of the specific IS activities and their management in accordance with the company strategy, as well as the evaluation and control of main lines of work in accordance with: the following information criteria: efficiency, effectiveness, availability, integrity, confidentiality, accord, safety and the financial and human resources through a flexible system of evaluation.

Keywords: Adapt to Changes, Input-Output Models, Effectiveness, Integrity, Principles

1 Introduction

When an organization introduces a change with a project or initiative, that change needs to be effectively managed on both the technical side and the people side. A technical side focus ensures that the change is developed, designed and delivered effectively. The discipline of project management provides the structure, processes and tools to make this happen. A people side focus ensures that the change is embraced, adopted and utilized by the employees who have to do their jobs differently as a result of the project. The discipline of change management provides the structure, processes and tools to make this happen. This new era requires a new breed of software, service and support solutions. The first step in the management journey begins with understanding and gaining control of your operations. It is critical to be able to visualize what is going on in your IT infrastructure and implement solutions that not only monitor, but deliver proactive management capabilities. Management software has traditionally focused on managing the elements and resources inside the data center or network operating center. Over time, those elements have become dispersed and distributed across wide networks. As the complexity of infrastructures increased, so did the need for management software to handle this complexity, anticipate problems and resolve them before they impact the business.

The managers need to understand the impact of the technology and of the informatics application upon the business they lead, taking into consideration the need to select the information in real time. Today's businesses are more and more organized, lead and automatized around the series of processes and of relations among them. These processes involve developments and lines of production and services, the administration of the interaction with the clients and the support of the processes and of the human resources. [3]

\section{Modern Principles of Change Manage- ment}

Here are some modern principles for organizational change management and effective employee training and development. [9] These ideas will not appeal to old-style paternalistic X-Theory organizations and cultures, unless they want to change for the better. These principles are for forward-thinking emotionally-mature organizations, who value integrity above results, and people above profit. 
The future is: Triple Bottom Line (Profit People Planet), Corporate Responsibility, Fair Trade, Sustainability, etc. - these are not just fancy words - they are increasingly and ever more transparently becoming the criteria against which modern successful organizations are assessed - by customers, employees, and the world at large. This is not to say that results and profit don't matter, of course they do. The point is that when you value integrity and people, results and profit come quite naturally. Some of these principles are:

- train or facilitate learning;

- organizational change;

- training;

- development;

- motivation.

\section{Common Problems in Project Manage- ment}

The purpose of this paper is to proving that an efficient system of evaluation of IS is an extremely efficient tool for the managers of project. [5] This tool can be an extremely useful informational support for the managers at every level of organization due to the:

- data's analyze;

- comparisons;

- measures;

- establishment of the main goals taking into account the impact they have on the organization and the risks involved.

A lot of problems are still to be solved in project management. Firstly, the architecture and performance should be considered together to make further improvements.

\section{Related Work}

In most information systems the management of databases is not considered to include neither explicit nor hidden inconsistencies. In real life situation information often come from different contradicting sources.

Software development projects require a lot of "paperwork" in the form of requirements documents, design documents, test plans, schedules, checklists, release notes, etc. It seems that everyone creates the documents from a blank page, from the documents used on their last project, or from one of a handful of high-priced proprietary software engineering template libraries. For those of us who start from a blank page, it can be a lot of work and it is easy to forget important parts.

\section{Role of the Individual in Successful Change}

Organizational change ultimately comes to life one person at a time [8]. Said another way, the individual is the unit of change. If changes are only successful when individuals do their jobs differently, then a structured approach for supporting individual change is central to projects delivering expected improvement [4]. In the end, change ultimately comes to life one individual at a time. Organizational change starts with an issue or opportunity that can come from a variety of places - strategic direction, annual planning, competitive landscape, customer demands, internal performance metrics, regulation, legislation, etc. [1]. Those issues or opportunities are then transformed into some sort of structured project or initiative - which can be accompanied by any number of tools like a charter, a schedule, a work breakdown structure, a budget, resource requirements, a timeline, etc. The project or initiative then cascades down to individual processes and behaviors - how specific individuals do their job and how the project or initiative impacts how they do their job. This is how change happens - issue or opportunity $\rightarrow$ project or initiative $->$ individual processes and behaviors. And ultimately it is individuals doing their jobs differently those results in a successful and sustainable change.

\section{Adapt to Changes}

The idea came up starting with the knowledge that the design of an IS begins with the identification of the problems and the opportunities in order to improve a business in an extremely complex economic system. [5] These problems and opportunities appear as a result of the desire of the companies to adapt to changes, a major aspect being occupied by informational processing. Due to the high degree of flexibility, the importance of the evaluation method is underlined. [2] 
Starting from the observation that a main characteristic of each level is to finalizing with a checkup and a validation in order to eliminate certain anomalies, it is underlined the fact that a good security of the IT and the administration practice's control of the complex IS projects is essential [4]. Under these circumstances, one can search new managerial solutions in order to integrate:

- time control;

- cost control;

- quality control of the working team;

- obtained results control.

One of the most important targets of the leadership is preventing and identification of the errors and frauds. The cost of the possible control measures must be compared with the probability of appearance of such fraud or error and the consequences.

The information are storage in a database in order to be able to make complex operations, the access to these information being made by taking into account the manager's purposes and abilities. The application becomes efficient through the design methods, allowing the interpretation and the manipulation of the data depending on the abilities of each user. One can describe such evaluating criteria able to understand the context, the user's medium and to respect the domain of the application [1].
The system can be adapted to each project manager's way of working, to the company's strategy, the financial and human resources offered by that company. The main criteria selections of the evaluation and control activities have been made after the following information criteria (Figure 1):

- effectiveness;

- integrity;

- confidentiality;

- availability;

- accordance;

- trust in the information.

The owner of the system is able to determine the level of adhesion to the control targets, whether as a self-evaluation, or as a reference related to a balance, or an independent cross - examination.

For every product, one can specify:

- the identification of the process;

- the purpose declaration for each process;

- the declaration of possibilities(how to keep the process under control in order to see if the target has been achieved)

- the IT resources;

- the criteria of information, by specifying the importance of the process for which is applied;

- the critical factors of success;

- the target indicators;

- the performance indicators.

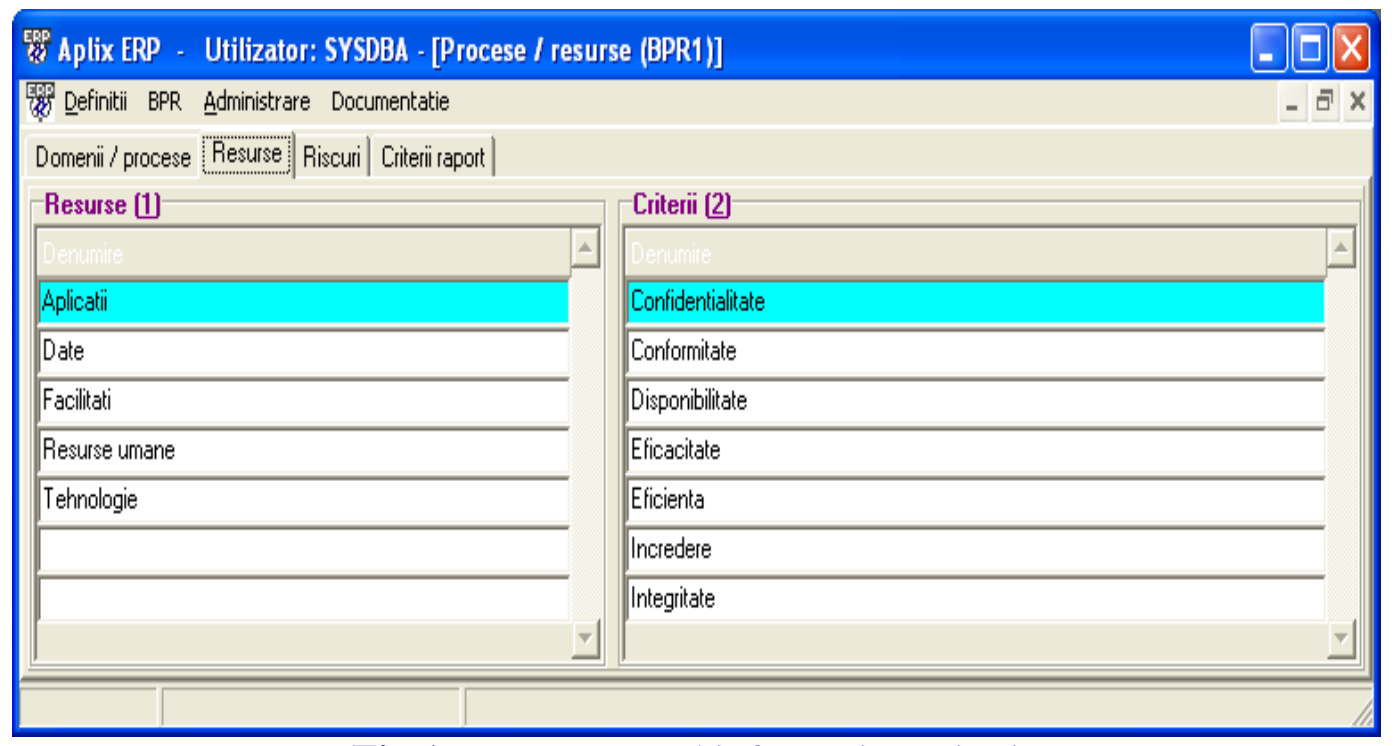

Fig.1. Resources and information criteria 
To all these we can add a great flexibility and adaptability to changes of the imposed system [8]. The role of the project's manager in planning, coordination and control of the complex IS project activities is being underlined [3].

It is a system, which allows the project's manager to establish the evaluation criteria and to concentrate upon the performances made in the administration of the specific IS activities.

The control of the activities is essential for the accomplishment of the projects. It is essential the role of each of the identified process in the Cobit standard for the accomplishment of the final project and the current stage of the organization, by grouping the processes in four activities:

- planning and organization of complex IS;

- the acquisition of goods and services for the project;

- delivering the designed system and the accomplishment of the paper documentation for the project;

- monitoring of complex IS.

The designed system is a necessary tool as much for the project's manager as well as for the economic unit's manager in order to obtain and maintain the competition advantage, to plan the necessary of information for the management of the technological changes, which should be one of the main goals of every company [7].

The need of a flexible evaluation system for the planning and organization of the activities taking place in complex IS projects for the acquisition and implementation, assistance and support, monitoring the performances of IS is being through this motivated. For the control of IS projects an evaluation method is being developed in order for each company to be able to self-evaluate the own performances regarding the IT. The principles are applied at informational, strategic, tactical and administrative level

The designed system allows the project manager to monitoring the activities and the costs, to evaluate periodically the progress made, to introduce some correction methods in order for the project to be applied to the managerial strategy, to be planned in accordance with the resources, to contribute to the realization of the purpose, to be practical.

A regular monitoring assures a dynamic reaction to the changes that appear, so that the project manager has pertinent information for the politics of the company's implementations.

It is highly important for the manager to establish reachable purposes, activities that are possible to administrate with the given resources.

Unrealistic deadlines, not wanted personnel and not working with the expert persons are reasons for which a projects fails.

The application is capable of interaction, of dividing the application as well as not synchronized interactions, divided space works [2].

The transformation of the company in terms of high level processes allows the managers to have a measure as far as the determination of the success of the processes and the comparison of their result with those of the competition. It is the ideal way to maintain a strong focus on the clients and their needs and to structure the relations with the partners and suppliers.

The processes supply a way to organize the IT's resources and to give priority to the IT's tasks as well. They represent the key which assures that the IT's investments support the strategies of the unit and guarantees a reasonable retrieve of the investments [7].

The designed system is a useful tool for the manager because it:

- offers a generalized frame of the processes of evaluation;

- is a way to evaluate and self-evaluate with flexible criteria;

- can serve as „best practice” for future researches.

The ethical values of one organization cannot be better than those of the employees (the leading positions are included) that create them, that make them work and supervise them! AND All this because there are some organizational factors that contribute to unauthorized actions such as: 
- the inefficiency of controls within the company;

- the inefficient decentralization of the report system;

- the penalizations of some employees that weren't announced to the entire company.
But the employees' ethical manner is not enough. Their abilities are another essential element of the control medium. The ability, that is knowledge and aptitudes necessary in every line of work, must be mentioned by the leaders. It is in the interest of every company to have the best employees. (Figure 2).

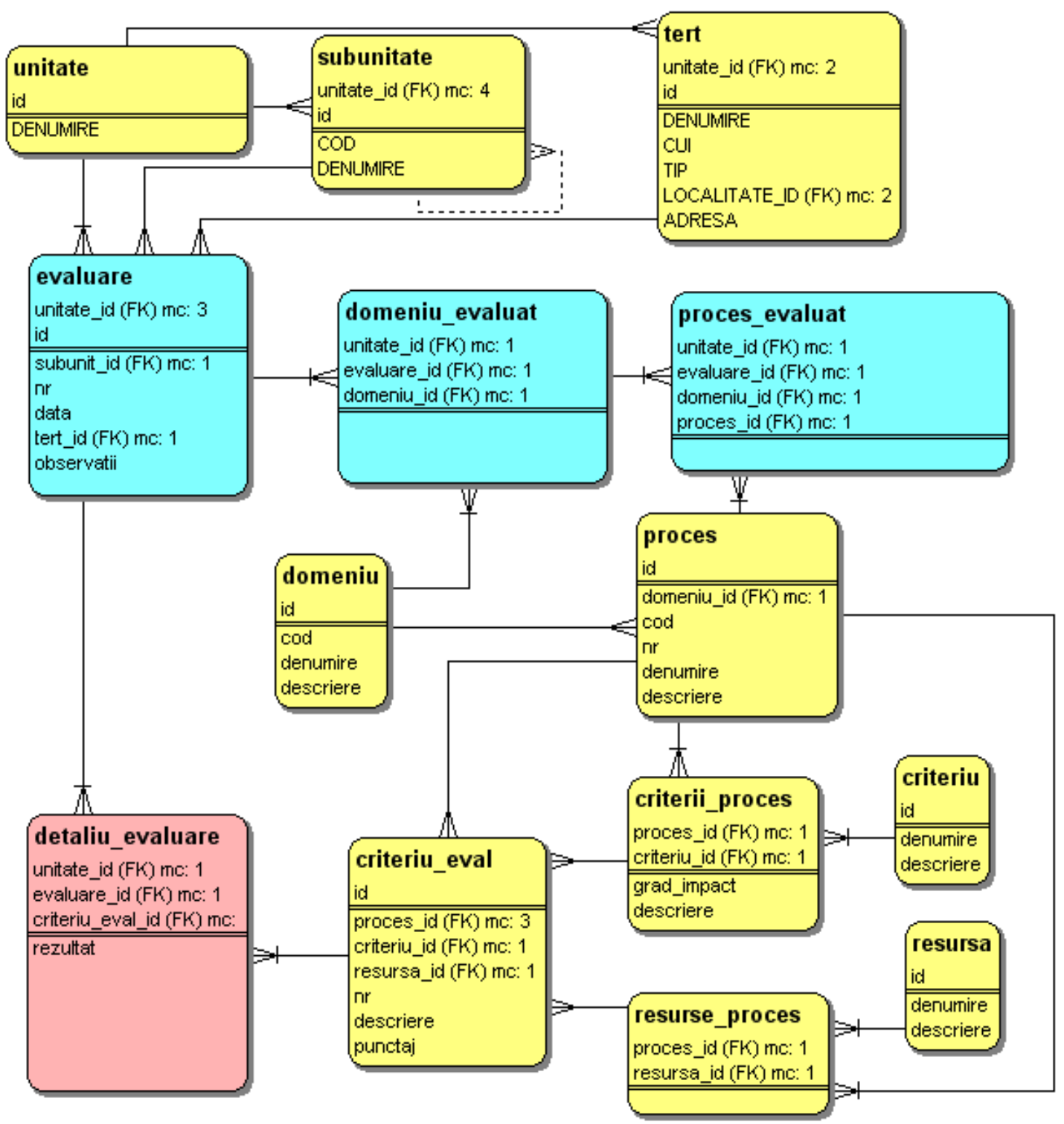

Fig. 2. Activities Diagram

Bosses and organizations still tend to think that people whom are managed and employed and paid to do a job should do what they're told to do. We are conditioned from an early age to believe that the way to teach and train, and to motivate people towards changing what they do, is to tell them, or persuade them. From our experiences at school we are conditioned to believe that skills, knowledge, and expectations are imposed on or 'put into' people by teachers, and later, by managers and bosses in the workplace.[9] But just because the boss says so, doesn't make it so. People today have a different perspective. And when you think about it, they're bound to.

Imposing new skills and change on people doesn't work because [9]: 
- it assumes that people's personal aims and wishes and needs are completely aligned with those of the organization, or that there is no need for such alignment;

- it assumes that people want, and can assimilate into their lives, given all their other priorities, the type of development or change that the organization deems appropriate for them.

Instead, organizations, managers, bosses and business owners would do better to think first about exploring ways to align the aims of the business with the needs - total life needs - of their people. Most people who go to work are under no illusion that their main purpose is to do what their manager says, so that the organization can at the end of the year pay outrageously high rewards to greedy directors, and a big fat dividend to the shareholders.

For complex changes, refer to the process of project management, and ensure that you augment this with consultative communications to agree and gain support for the reasons for the change. Involving and informing people also creates opportunities for others to participate in planning and implementing the changes, which lightens your burden, spreads the organizational load, and creates a sense of ownership and familiarity among the people affected [9].

\section{People React Differently to Change}

Motivation, management, communications, relationships - focused on yourself or others are a lot more effective when you understand yourself, and the people you seek to motivate or manage or develop or help [9]. Motivation, management, communications, relationships - focused on yourself or others - are a lot more effective when you understand yourself, and the people you seek to motivate or manage or develop or help.

The personality theories that underpin personality tests and personality quizzes are surprisingly easy to understand at a basic level. This section seeks to explain many of these personality theories and ideas. This knowledge helps to develop self-awareness and also to help others to achieve greater self-awareness and development too.
Developing understanding of personality typology, personality traits, thinking styles and learning styles theories is also a very useful way to improve your knowledge of motivation and behavior of self and others, in the workplace and beyond. Understanding personality types is helpful for appreciating that while people are different, everyone has a value, and special strengths and qualities, and that everyone should be treated with care and respect [9].

Completing personality tests with no knowledge of the supporting theories can be a frustrating and misleading experience - especially if the results from personality testing are not properly explained, or worse still not given at all to the person being tested. Hopefully the explanations and theories below will help dispel much of the mystique surrounding modern personality testing.

There are many different personality and motivational models and theories, and each one offers a different perspective [9]. The more models you understand, the better your appreciation of motivation and behavior. For organizational change that entails new actions, objectives and processes for a group or team of people, use workshops to achieve understanding, involvement, plans, measurable aims, actions and commitment. Encourage your management team to use workshops with their people too if they are helping you to manage the change [9].

You should even apply these principles to very tough change like making people redundant, closures and integrating merged or acquired organizations. Bad news needs even more careful management than routine change. Hiding behind memos and middle managers will make matters worse. Consulting with people, and helping them to understand does not weaken your position - it strengthens it. Leaders who fail to consult and involve their people in managing bad news are perceived as weak and lacking in integrity. Treat people with humanity and respect and they will reciprocate. 
8 Problems and the Opportunities in Order to Improve a Business in an Extremely Complex Economic System

These problems and opportunities appear as a result of the desire of the companies to adapt to changes, a major aspect being occupied by informational processing. Due to the high degree of flexibility, the importance of the evaluation method is underlined. Starting from the observation that a main characteristic of each level is that of finalizing with a checkup and a validation in order to eliminate certain anomalies, it is underlined the fact that a good security of the IT and the administration practice's control of the complex IS projects is essential. Under these circumstances, one can search new managerial solutions in order to integrate:

- time control;

- cost control;

- quality control of the working team;

- obtained results control.

To all these we can add a great flexibility and adaptability to changes of the imposed system. The role of the project's manager in planning, coordination and control of the complex IS project activities is being underlined. The designed system is a necessary tool as much for the project's manager as well as for the economic unit's manager in order to obtain and maintain the competitional advantage, to plan the necessary information for the management of the technological changes, which should be one of the main goals of every company.

The need of a flexible evaluation system for the planning and organization of the activities taking place in complex IS projects for the acquisition and implementation, assistance and support, monitoring the performances of IS is being through this motivated. For the control of IS projects an evaluation method is being developed in order for each company to be able to self-evaluate the own performances regarding the IT. The principles are applied at informational, strategically, tactical and administrative level.

The designed system allows the project manager to monitoring the activities and the costs, to evaluate periodically the progress made, to introduce some correction methods in order for the project to be applied to the managerial strategy, to be planned in accordance with the resources, to contribute to the realization of the purpose, to be practical.

A regular monitorization assures a dynamic reaction to the changes that appear, so that the project manager has pertinent information for the politics of the company's implementations. It is highly important for the manager to establish reachable purposes, activities that are possible to administrate with the given resources. Unrealistic deadlines, not wanted personnel and not working with the expert persons are reasons for which a projects fails. The informatics solution, which is given underlines the functionality and the easy way to handle it. The application is capable of interaction, of dividing the application as well as not synchronized interactions, divided space works. The transformation of the company in terms of high level processes allows the managers to have a measure as far as the determination of the success of the processes and the comparison of their result with those of the competition. It is the ideal way to maintain a strong focus on the clients and their needs and to structure the relations with the partners and suppliers. The processes supply a way to organize the IT's resources and to give priority to the IT's tasks as well. They represent the key which assures that the IT's investments support the strategies of the unit and guarantees a reasonable retrieve of the investments.

The designed system is an useful tool for the manager because it:

- offers a generalized frame of the processes of evaluation;

- is a way to evaluate and self-evaluate with flexible criteria;

- is an alternative to the complex analysis of the evaluation results;

- can serve as „best practice” for standardizations and future researches.

Most of the economic decisions are based upon financial information. However, as decident, to have information at the right time is not everything. These information must be trustable. The need for competent and inde- 
pendent evaluations is bigger every day. The given evaluation system is a tool that allows:

- the check up in order to see if the necessary conditions are fulfilled to assure the equilibrium of an organization;

- to control the disorder, to adapt to changes;

- to evaluate the degree of security and the risks undertaken by a company.

This kind of IS evaluation is very useful. The aims of every institution and the manner, in which they are reached, depend on the abilities and leading manner of the managers. Their integrity and attachment as far as the ethical values are concerned are reflected by their actions and judgments. The ethical values of one organization cannot be better than those of the employees (the leading positions are included)that create them, that make them work and supervise them! AND All this because there are some organizational factors that contribute to fraudulent actions such as:

- the inexistence and inefficiency of controls within the company;

- the excessive and inefficient decentralization of the report system;

- the penalizations of some employees that weren't announced to the entire company.

But the employees' ethical manners are not enough. Their abilities are another essential element of the control medium. The ability, that is knowledge and aptitudes necessary in every line of work, must be mentioned by the leaders. It is in the interest of every company to have the best employees.

\section{The Managerial Philosophy and the Leading Manner}

We can include in this category factors such as: taking the risks, instituted politics and procedures, giving responsibilities, the attitude in regard of financial reports. The control medium is strongly influenced by the degree in which the employees know their role in reaching the company's targets. In the end, the organizational structure represents the general frame where the necessary activities will be planned, executed, controlled in order to reach the targets. Most often, she is the result of the managerial philosophy and can be centralized, decentralized and structured on a functional base, in comparison with the size of the company and the nature of its activities.

The company must be aware of the risks and must be able to face them. The activities and the risks involved by these activities must be evaluated in such a manner as to allow the company to take only the risks it can afford and in order to avoid the useless ones. But one of the prime conditions in risk evaluation is to establish some well-defined and compatible targets with the company's evolution. The risks evaluation assumes the identification and analyze of those events that can interfere in the reach of those purposes.

After being identified, all the risks are being evaluated. Often enough, this process, which can be more or less formal, includes:

- the evaluation of the importance of every risk;

- the evaluation of the probability or the frequency of risks appearance;

- the actions that must be taken in order to prevent the risks and the costs involved in these actions.

After evaluating the risks, the next step, every company should take, is, on one hand, establishing the procedures of control and, on the other hand, establishing the activities which are about to be controlled. The politics and the procedures of the company must be reviewed in such a manner in which to assure an effective leadership. These sort of activities must exist at all levels and for all the company's positions: agreements, authorizations, check-ups, reviews, performance evaluation, the separation of job tasks. (Figure 3). The communication must be done in all directions: from up - down (from the leaders to the employees), from down- up (from the employees to the leaders) or separating the information at the same level.

One of the most important targets of the leadership is preventing and identification of the errors and frauds. The cost of the possible control measures must be compared with the probability of appearance of such fraud or error and the consequences. The evaluation 
system offers the leaders the required data necessary for the administration of that busi- ness. But it's still the job of those being in charge to create and launch such a system.

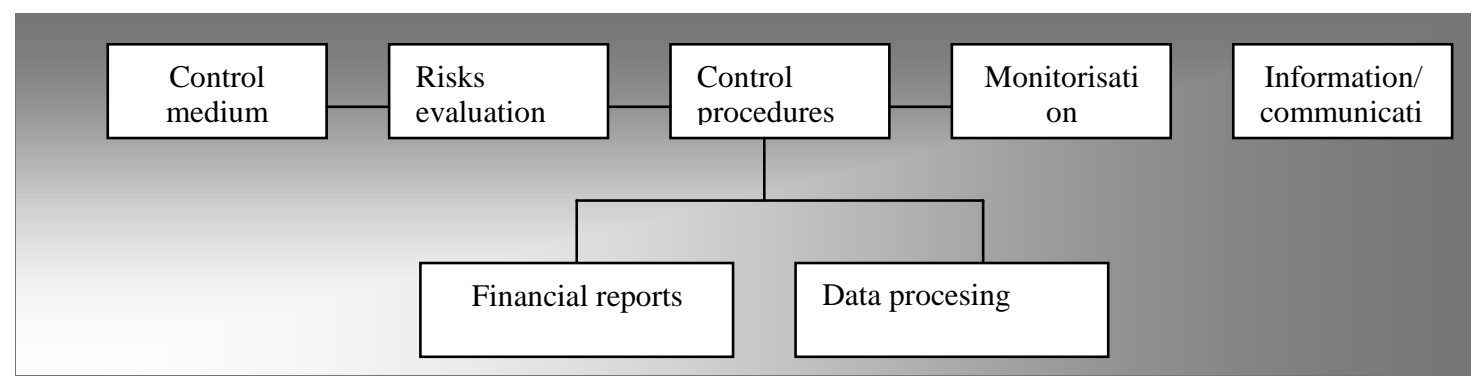

Fig.3. Types of controlled activities

The owner of the system is able to determine the level of adhesion to the control targets, whether as a self-evaluation, or as a reference related to a balance, or an independent cross - examination.

For every product, one can specify (Figure 4):

- the identification of the process;

- the purpose declaration for each process
- the IT resources;

- the criteria of information, by specifying the importance of the process for which is applied;

- the critical factors of success;

- the target indicators;

- the performance indicators.

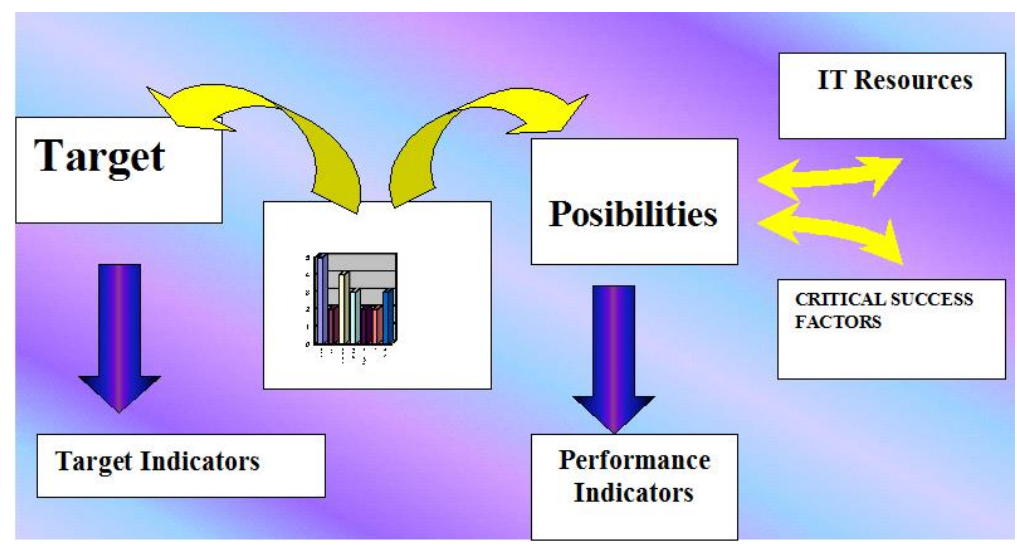

Fig.4. The elements of a process

This evaluation tool can become for the company:

- a self-evaluation method which decides where is the company situated;

- a method for the use of the results of the evaluation in order to establish the targets for the company's future development, based upon the level wished to be reached, which does not necessarily implies level 5 ;

- a planning method for the reach of the desired purposes;
- a method to establish the priorities in the development of the projects, based on a classification of the projects and an analyze of their benefits as compared to the costs.

For every criteria of evaluation, the company must use a measurement scale between 0 and 5 , in order to define the estimated position. This can be easily compared with the other three targets: target performance international standards and the best practice. One must mention the fact that there is a difference between the measurement of the abilities and 
that of performances. For example, the achievement of the abilities for a certain security or control of practices is one of that decisions that must be taken and done, but the consistent appliance of the abilities, once required, also demands to be measured.

The purpose in choosing the main projects is that of identification of those projects where one can have quick results and benefits. The most suitable candidates for quick benefits are usually those where the discrepancies are smaller and the closing price and the failure risks are also smaller, and the impact upon the benefits of the business is higher (Figure 5).

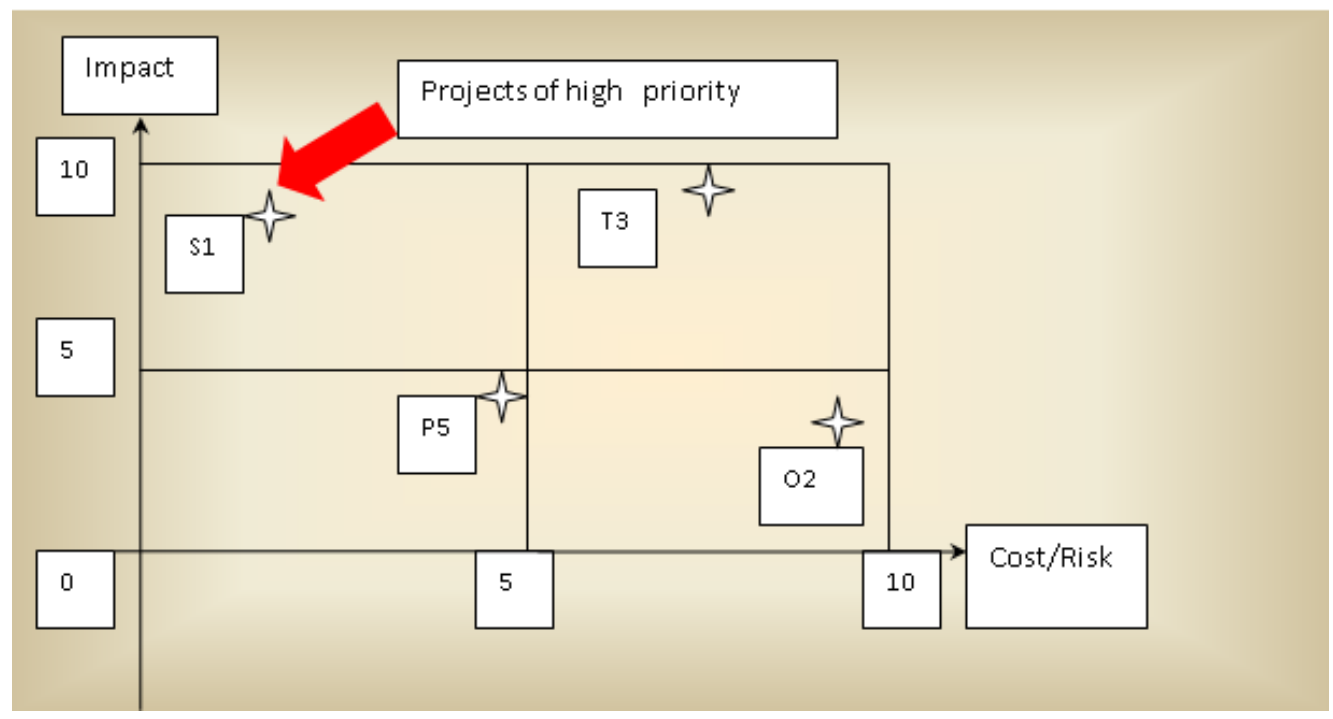

Fig. 5. The position in relationship with the impact on the business and risk

The manner in which the priority is being attributed to the projects is through the identification of the rapid benefits that can be achieved. The best candidates for rapid benefits are usually those projects in which the gaps are smaller, where the costs to close a gap are as small as possible, where the risk of failure and the impact of the benefits on the business is higher. The projects could be evaluated for impact and cost/risk on a scale from 0 to 10 for each of these variables. The projects can be pointed on a graphic that can become a support tool for the decision of the manager, showing the relative impact and the costs/risks. The projects that have a bigger impact and a relatively small price are better candidates as well as quicker winners. The effective administration of the IT has a major importance in the success and survival of the company.

For many companies, the data and the technology they have, represent the biggest values. Indeed, the information and the information systems are general for the entire company - from the users platform to the lo- cal networks and those of bigger cover, to the servers of the user within the computers. Many companies recognize the potential benefits produced by technology. Yet, the successful companies understand this and administrate the associated risks with the introduction of new technologies. Thereby, the administration needs a good appreciation and a basic understanding of the risks and limitations within the IT in order to supply adequate controls.

The companies are being reorganized with the modernization processes and are using the IT's investments to improve their competitional position. The reorganization of the business, the correct size, the external sources, the authorization, are all changes that come to interact with the manner in which the companies and the governmental organizations work. These changes have and will continue to have other major impacts for the administration and the operational control structures within one company.

An evaluating system based on the four mentioned activities in the Cobit standard of IT.'s 
evaluation is extremely useful or the project manager in order to control:

- the planning and organization processes;

- the implementation processes;

- the assistance and support processes;

- monitoring processes.

\section{Conclusions}

A successful management is based upon efficient communication. The evaluation of the assistance and support activity is based upon the defining process of the levels of service, the leading of the service team, the performance management, of offering a continuous service, the security of the system, the identification of the costs for assistance and support, the teaching and training of the users, the assistance and advise of the clients, the configuration management, the organization of the problems and incidents that might appear, the data , facilities and operation management( addition, accuracy, validation, upgrading and stocking). All the attempts in defining the leadership is centered upon the idea that the leadership is different from the management, that leaders are different from the managers, due to certain personal qualities and abilities. In a corporation, an efficient leader has a future strategy of the organization, which takes into consideration the true interests on a long term of all those involved in it Then the leader develops a strategy in order to reach that purpose by obtaining support from the employees and in the same time motivating them to reach that purpose.

Good managers realize that the recognition of the symptoms of the problems, the diagnosis of these problems and then the confrontation are imperative solutions for the business if we want the business to work in the most proper way.

Problems come out in different ways. The Feedback can offer information regarding the gap between actual performance and the desired one. We could say that feedback highlights the problems. Also the external feedback is extremely important and must not be ignored..

The three most relevant and key elements in order for the project to be realized are:

- technical feasibility - added to the present system, available technology for the users requests;

- economic feasibility -the period of time in which a project is designed, the cost for its planning, the cost for the employees' time of study, the estimative cost of the hardware and software equipment and their development;

- operational feasibility - The well function of the system after it has been installed, the usage of the designed project.

For the approval of each project there must be identified needs at first. It is also necessary that the project is possible from the technical point of view. The question is if the present technology allows its accomplishment, and if these technologies exist, are they accessible taking into consideration the knowledge, the abilities, the budgets, the human and material resources..

The project manager is responsible for the correct investment of the resources, as well as for their usage in order to obtain the desired result.

The demand for the management teams is to think in terms of process and not in divisions and functional units if the companies want to successfully compete among them, they must frequently modify the processes, to adapt them to the market requests and to add new tools which have to adapt to these changes.

Taking the decisions without a proper subversion of their well function has usually no effect whatsoever. It is the same in the planning activity which will bring lesser benefits if the planned activities are not supervised and controlled. The control implies a continuous evaluation of the progresses made in the process of achievement of the project, as compared with certain criteria. Generally, these criteria are time, quality and budget. The degree of complexity of the project is a factor, which determine the report and control method. A complex project necessarily requires intermediate levels of leadership and report. 


\section{References}

[1] C. Cârstea, N. David "Solutions about Evaluation and Control Data For Complex Information Systems", pag.165-169, A4, WSEAS Conference in Istanbul, Turkey, 7th International Conference on TELEINFO'08, New Aspects of Telecommunications and Informatics TELEINFO '08, 27-30 may 2008, ISBN-978960-6766-64-0, ISSN 1790-5117

[2] Gh. Sabau, A. Cretan, "A Negotiation Approach for Inter-Organizational Alliances", International Conference on Future Networks, Proceedings, 2009

[3] Gh. Sabau, M. Muntean, A. R. Bologa, R. Bologa, "Analysis of Integrated Software Solutions Market for Romanian Higher Education", Journal of Economic Computation and Economic Cybernetics Studies and Research, 2009, pp. 197-203,
ISSN $0424-267 \mathrm{X}$;

[4] J. Sandberg, "Understanding Human Competences at Work: An Interteprative Approac", The Academy of Management Journal, Vol.43, No 1, 2005

[5] C. Carstea, Asistarea deciziei in managementul proiectelor informatice, Editura Economica, 2010, ISBN 978-973709-377-6

[6] A. Laufer \& E. Hoffman, "Project Management Success Stories: Lessons of Project Leaders", New York: John Wiley \& Son, 2000, ISBN: 0-471-36007-4

[7] C. Olinger, "The Issues Behind ERP Acceptance and Implementation", APICS: The Performance Advantage , 2007.

[8] http://www.change-management.com

[9] http://www.businessballs.com/organizatio nalchange.htm

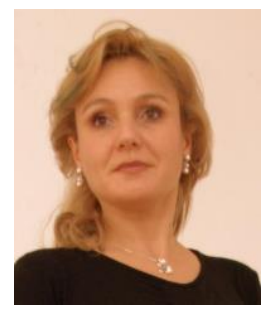

Claudia CARSTEA is Associate Professor in Databases and Management Information Systems at "George Baritiu" University, Brasov, Romania. Holds a $\mathrm{PhD}$ diploma in Cybernetics, Statistics and Economic Informatics, from 2008 at Bucharest Academy of Economic Studies, Romania. Research activities focus in the fields of: performance management and measurement in design information systems, business networks, databases, risk evaluation in project management, process control in management information systems. In the present is Vice-Rector of "George Baritiu" University of Brasov, Romania. She is author and co-author of more than 80 research papers published in referred journals and conference proceedings, and he has been author and editor more than 12 national and international books.

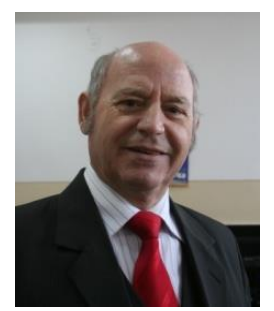

Gheorghe SABAU is a graduate of Babes-Bolyai University of Cluj, Romania. He graduated from the faculty of Economic Studies in 1968. He got the title of doctor in economy in the economic informatics specialization in 1982. Since 1993 he has been directing the doctoral activity of those who train to get the title of "doctor". At present he is a professor with the department of the faculty of Economic Informatics of the Faculty of Cybernetics, Statistics and Economic Informatics of the Academy of Economic Studies of Bucharest. He carried documentary and specialization activity with the University of Reading (England), Stanford University of USA, University of Paris, University of Athens. His domains of interest are Information systems and Databases. Among the books he published are: "Economic Informatics" (1987), "The Cobol language" (1989), "Databases Practice", volume 1 and 2 (1989), "Informatics Systems and Databases" (1998), "Information Systems - Analysis, Design and Implementation" (2003) and "Databases" (2008). 